\title{
Measurement of drug diffusivity in stratum corneum membranes and a polyacrylate matrix *
}

\author{
Achim Göpferich and Geoffrey Lee \\ Institute for Pharmaceutical Technology and Biopharmaceutics, Heidelberg University, Heidelberg (Germany)
}

(Received 5 July 1990)

(Modified version received 4 September 1990)

(Accepted 25 September 1990)

Key words: Stratum corneum; Polymer matrix; Diffusion; Measurement

\begin{abstract}
Summary
Diffusivities have been determined for the drug clenbuterol for three non-sink, diffusional problems: diffusion out of a polymer matrix, diffusion through excised, human stratum corneum, and the combined case of diffusion out of a polymer matrix through contiguous, excised stratum corneum. Numerical solution of the diffusion equation for these problems yields theoretical values of drug mass in the acceptor phase, $m_{\mathrm{a}}(t)_{\mathrm{th}}$, vs time. Diffusion was then measured experimentally and the best value for diffusivity obtained by comparing the values of drug mass in the acceptor phase, $m_{\mathrm{a}}(t)_{\text {exp }}$, with the corresponding $m_{\mathrm{a}}(t)_{\mathrm{th}}$ data. In this fashion it was possible to obtain diffusivities for diffusion out of the polymer matrix for various values of drug loading and matrix thickness. For the problem of diffusion through stratum corneum a value for the partition coefficient of clenbuterol as well as for its diffusivity could be determined. The two diffusivities obtained for the combined model (i.e., $D_{\mathrm{m}}$ within the matrix and $D_{\mathrm{s}}$ within the stratum corneum) agree quite well with those obtained for the two separate problems, but are more scattered. Partition coefficients could not be accurately determined for diffusion out of the matrix or the combined problem.
\end{abstract}

\section{Introduction}

This study originated from measurements of drug diffusion out of a polymer matrix through a contiguous membrane of excised stratum corneum into a well-stirred acceptor medium. We wished to determine the effects of the properties of the matrix on the diffusional behaviour of the drug within

\footnotetext{
* Dedicated to Professor Helmut Stamm on the occasion of his 65 th birthday.
}

Correspondence: Geoffrey Lee, Pharmazeutische Technologie und Biopharmazie, Im Neuenheimer Feld 366, D-6900 Heidelberg, Germany. each layer of this bilayer system. To avoid intrinsic error we considered realistic experimental and boundary conditions, thereby recognising, for example, the removal of samples from the system for analysis and the existence of a non-sink. This diffusional problem is related to a non-steady state model analysed by Guy and Hadgraft (1980) for percutaneous absorption with release into a sink in vivo. The analysis of such multi-layer problems is, however, associated with complications arising from the inversion problem for the Laplace transforms used as a solution method. These complications ultimately led Guy and Hadgraft to adopt a compartmental model to represent percutaneous absorption in vivo, with diffu- 
sivities being replaced by rate constants (Guy et al., 1982). Similar difficulties arise with the analytical solution to the non-sink, non-steady state diffusional problem being considered here: it is not readily expressed in a form convenient for computation (see Appendix). The compartmental approach would not be a suitable alternative, as such a model would not directly relate the specific properties of a matrix-type delivery device (drug loading, matrix thickness and drug diffusivity within the matrix) to the rate of appearance of drug on the inner side of the stratum corneum. The problem is better tackled numerically, giving an easily-manipulated, algebraic solution to Fick's Second Law. We wished to determine if this numerical solution could be used to calculate diffusivity in the matrix and stratum corneum from in vitro experimental data obtained for various matrix properties. As this is not necessarily a straightforward task for a multilayer system we first separated the original bilayer model into its two components problems, namely, non-sink diffusion out of a rectangular matrix (analytical solution: Crank, 1975) and non-sink diffusion through a plane membrane (analytical solution: Spacek and Kubin, 1967). Experimental data were determined for each of these problems, to which the theoretical diffusional behaviour predicted by the corresponding numerical solution were fitted. Values for diffusivity and partition coefficient could thereby be obtained.

\section{Materials and Methods}

\section{Measurement of diffusion}

For each of the three problems the diffusion of the water-soluble drug clenbuterol (Mol. Wt. 277 and $\mathrm{p} K_{\mathrm{a}}$ 9.5) was measured at $35^{\circ} \mathrm{C}$ using an isotonic, $\mathrm{pH} 8$ phosphate buffer solution as receptor/donor phase:<smiles>CC(C)(C)N=CC(O)c1cc(Cl)c(N)c(Cl)c1</smiles>

Polymethylacrylate-ester matrices (freeze-dried Eudragit NE30D, Röhm Pharma, Weiterstadt, Germany) of various thickness and containing various concentrations of drug were prepared on a

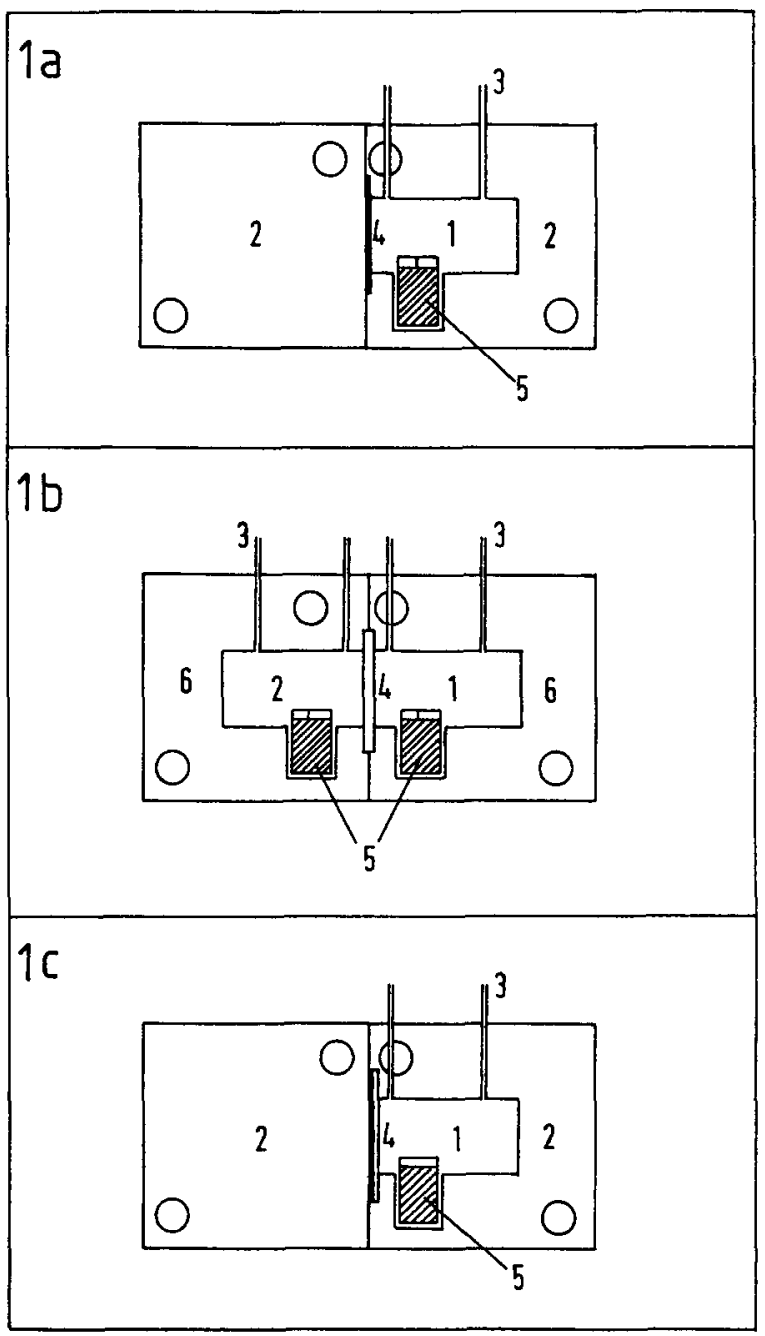

Fig. 1. The glass diffusion cell. (a) Construction for measurement of diffusion out of polymer matrix: (1) acceptor chamber $\left(V_{\mathrm{a}}=4 \mathrm{ml}\right)$; (2) water jacket $\left(35^{\circ} \mathrm{C}\right)$; (3) sampling port; (4) polymer matrix ( $A=1 \mathrm{~cm}^{2}$ ); (5) magnetic stirrer (600 rpm). (b) Construction for measurement of diffusion through excised stratum corneum: (1) donor chamber $\left(V_{\mathrm{d}}=4 \mathrm{ml}\right)$; (2) acceptor chamber ( $\left.V_{\mathrm{a}}=4 \mathrm{ml}\right)$; (3) sampling port; (4) stratum corneum $\left(A=1 \mathrm{~cm}^{2}\right)$; (5) magnetic stirrers $(600 \mathrm{rpm}) ;(6)$ water jacket $\left(35^{\circ} \mathrm{C}\right) .(\mathrm{c})$ Construction for measurement of diffusion out of polymer matrix through excised stratum corneum: (1)-(3) and (5) as for panel a; (4) polymer matrix with attached stratum corneum. 
TABLE 1

Initial and boundary conditions for Eqn 1

(1) Diffusion out of a matrix

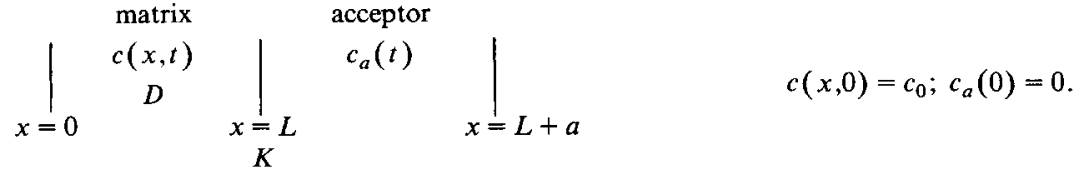

$$
\begin{aligned}
& x=0\left[\mathrm{~B}^{*}\right]: \frac{\partial c(0, t)}{\partial x}=0, \quad t>0 ; \quad x=L\left[\mathrm{~F}^{*}\right]: \frac{a \cdot \mathrm{d} c_{\mathrm{a}}(t)}{K \cdot \mathrm{d} t}+D \frac{\partial c\left(L_{\mathrm{m}}, t\right)}{\partial x}=0, \quad \ldots
\end{aligned}
$$

(2) Diffusion through stratum corneum

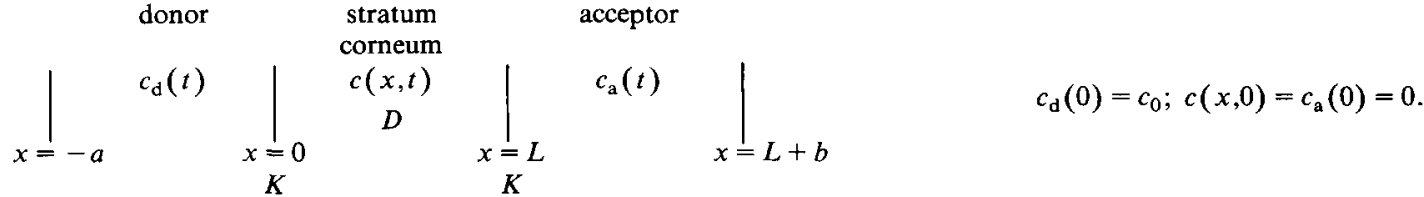

$$
\begin{aligned}
& x=0\left[\mathrm{~F}^{*}\right]: \frac{a \cdot \mathrm{d} c_{\mathrm{d}}(t)}{K \cdot \mathrm{d} t}+D \frac{\partial c(0, t)}{\partial x}=0, \quad t>0 ; \quad x=L\left[\mathrm{~F}^{*}\right] ; \frac{b \cdot \mathrm{d} c_{\mathrm{a}}(t)}{K \cdot \mathrm{d} t}+D \frac{\partial c\left(L_{\mathrm{s}}, t\right)}{\partial x}=0, \quad t>0
\end{aligned}
$$

(3) Combined problem
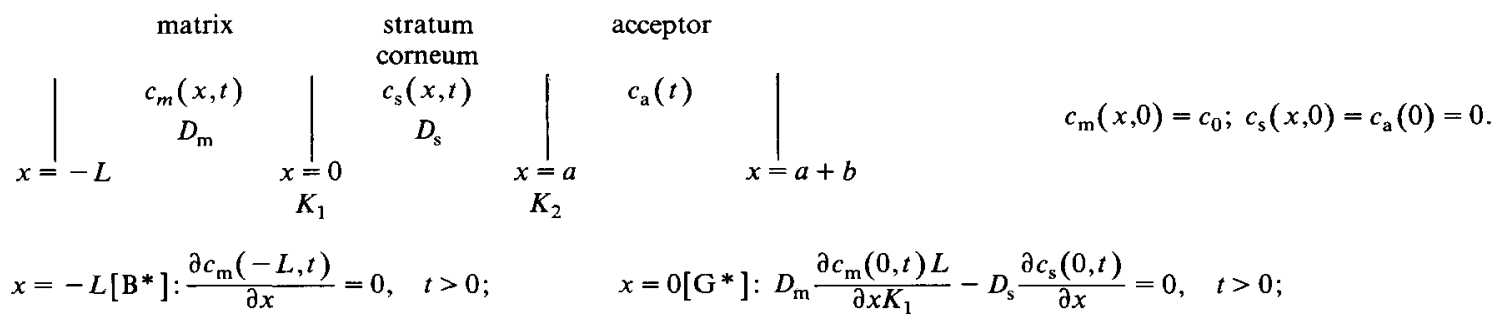

$x=a\left[\mathrm{~F}^{*}\right]: \frac{b \cdot \mathrm{d} c_{\mathrm{a}}(t)}{K_{2} \cdot \mathrm{d} t}+D_{\mathrm{s}} \frac{\partial c_{\mathrm{s}}(a, t)}{\partial x}=0, \quad t>0$.

* Classification according to Carslaw and Jaeger (1959b).

siliconised paper base by solvent evaporation (Zierenberg, 1985). Stratum corneum membranes were prepared from whole human skin excised from the inner thighs of cadavers within $48 \mathrm{~h}$ post-mortem (Kligmann and Christophers, 1963). The thickness of each matrix or piece of stratum corneum was measured at 20 positions using an Elcometer (Elcometer Instruments, Manchester, U.K.)

The diffusion of the drug out of the polymer matrix was measured by affixing the latter in a glass diffusion cell as illustrated in Fig. 1a. Samples of the acceptor solution were removed at regular intervals, divided into three aliquots and each individually assayed by HPLC. Drug diffusion through excised, human stratum corneum membranes was determined with the cell construction shown in Fig. $1 \mathrm{~b}$ and using a method described by Swarbrick et al. (1984). Drug diffusion out of the matrix through excised, human stratum corneum was measured with the cell arrangement shown in Fig. 1c. Care was taken to ensure asnear-as-possible complete contact between the outer matrix surface and the inner side of the contiguous stratum corneum. Each experiment was performed at least four times and the results rep- 
resented as plots of the experimentally determined mass of drug in the acceptor solution, $m_{\mathrm{a}}(t)_{\text {exp }}$, vs time, $t$. The relative error in the HPLC-determination of $m_{\mathrm{a}}(t)_{\exp }$ was found to be $<1 \%$, even for the smallest amounts determined in the receiving chamber at short times.

\section{Calculation of diffusivity}

Diffusivities for the drug were calculated for each of the three problems by fitting the theoretical values of $m_{\mathrm{a}}(t)_{\mathrm{h}}$ to the corresponding experimentally determined values of $m_{\mathrm{a}}(t)_{\mathrm{exp}}$. The former were obtained using a finite-difference method (Crank and Nicolson, 1947; Smith, 1987) to approximate numerically the diffusion equation for the linear movement of a drug with constant diffusivity, $D$, through a finite plane sheet:

$D \cdot c(x, t)_{x x}-c(x, t)_{t}=0$.

$c$ is the drug concentration, $x$ the space coordinate, and $t$ the time, with subscripts denoting partial derivatives. We thus assume isotropic phases and spontaneous partitioning at boundaries. For each of the three problems a threediagonal matrix comprising Eqn 1 in finite difference form together with the corresponding initial and non-sink boundary conditions (summarised in Table 1) was solved by a programme in Pascal on an Epson PC AX3 personal computer with an $80386 / 80387$ processor/co-processor. Solution by Gauss's elimination method yielded the theoretical concentration profile of the drug within the sheet, $c(x, t)_{\text {th }}$, from which the theoretical mass in the acceptor solution, $m_{\mathrm{a}}(t)_{\mathrm{th}}$, was obtained as a function of time. All experimental conditions capable of influencing the diffusion rate were taken into account in the calculation, for example, the removal of samples from the acceptor chamber of the diffusion cell. The sum of the accumulated formula and rounding-off errors was assessed from $m_{\mathrm{a}}(48 \mathrm{~h})_{\text {th }}$ and found to be $<1.5 \%$. The $m_{\mathrm{a}}(t)_{\mathrm{h}}$ values were then fitted to the $m_{\mathrm{a}}(t)_{\exp }$ values using the improved simplex method of Nelder and Mead (1967) to yield the best values for the diffusivity, $D$, and the partition coefficient, $K$. A smooth curve was then drawn through the coordinates of $m_{\mathrm{a}}(t)_{\mathrm{th}}$ using polynomial splines of third degree.

\section{Results and Discussion}

\section{Diffusion out of polymer matrix}

Fig. $2 a$ and $b$ shows results for the effect of drug loading $\left(m_{\infty}\right)$ and matrix thickness $(L)$ on the coordinates of $m_{\mathrm{a}}(t) \exp$ plotted vs $t$ for diffusion out of the polymer matrix. The curves passing through the coordinates are the best fits of $m_{\mathrm{a}}(t)_{\mathrm{th}}$ to $m_{\mathrm{a}}(t)_{\exp }$. The increased release rates seen with either greater drug loading or greater matrix thickness are in agreement with curves generated from the analytical solution to this non-sink problem (Crank, 1975). Since the matrix used to determine $m_{\mathrm{a}}(t)_{\mathrm{th}}$ was non-singular it was possible to determine $D$ unequivocally from the fitting of $m_{\mathrm{a}}(t)_{\text {th }}$ to $m_{\mathrm{a}}(t)_{\text {ex. }}$ Table 2 shows these diffusivities as mean values with standard deviations. For a constant matrix thickness of $45.2 \mu \mathrm{m}$ mean diffusivity increases with greater drug loading, as already observed by Zierenberg (1985) for the same polymer. As to be expected, there is no clear influence of matrix thickness on diffusivity, and the scatter observed must be due to variation in the polymer system itself or error in the method. The accuracy of the method depends on the HPLC-analysis error $(<1 \%)$, the accumulated formula and rounding-of errors $(<1.5 \%)$, and the errors in the measurement of $L$ (approx. $2 \%$ ) and $m_{\infty}$ ( $<2 \%$, when determined from the exact composition of the polymer/drug/solvent solution used to prepare a matrix of known weight). The effects of these errors alone are not of sufficient magnitude to be responsible for the observed scatter in diffusivity. The use of Eqn 1, however, assumes constant diffusivity within an isotropic medium, both of which may be questionable conditions for this system.

It was not possible to determine an unequivocal partition coefficient, the values obtained showing either great variation or being unrealistic (Table 2 ). This results from the strongly non-linear influence of $K$ on the diffusion rate, $\mathrm{d} m_{\mathrm{a}}(t) / \mathrm{d} t$, as illustrated theoretically by the dimensionless plots of $m_{\mathrm{a}}(t)_{\mathrm{th}} / m_{\infty}$ vs $D t / L^{2}$ for this problem in Fig. 

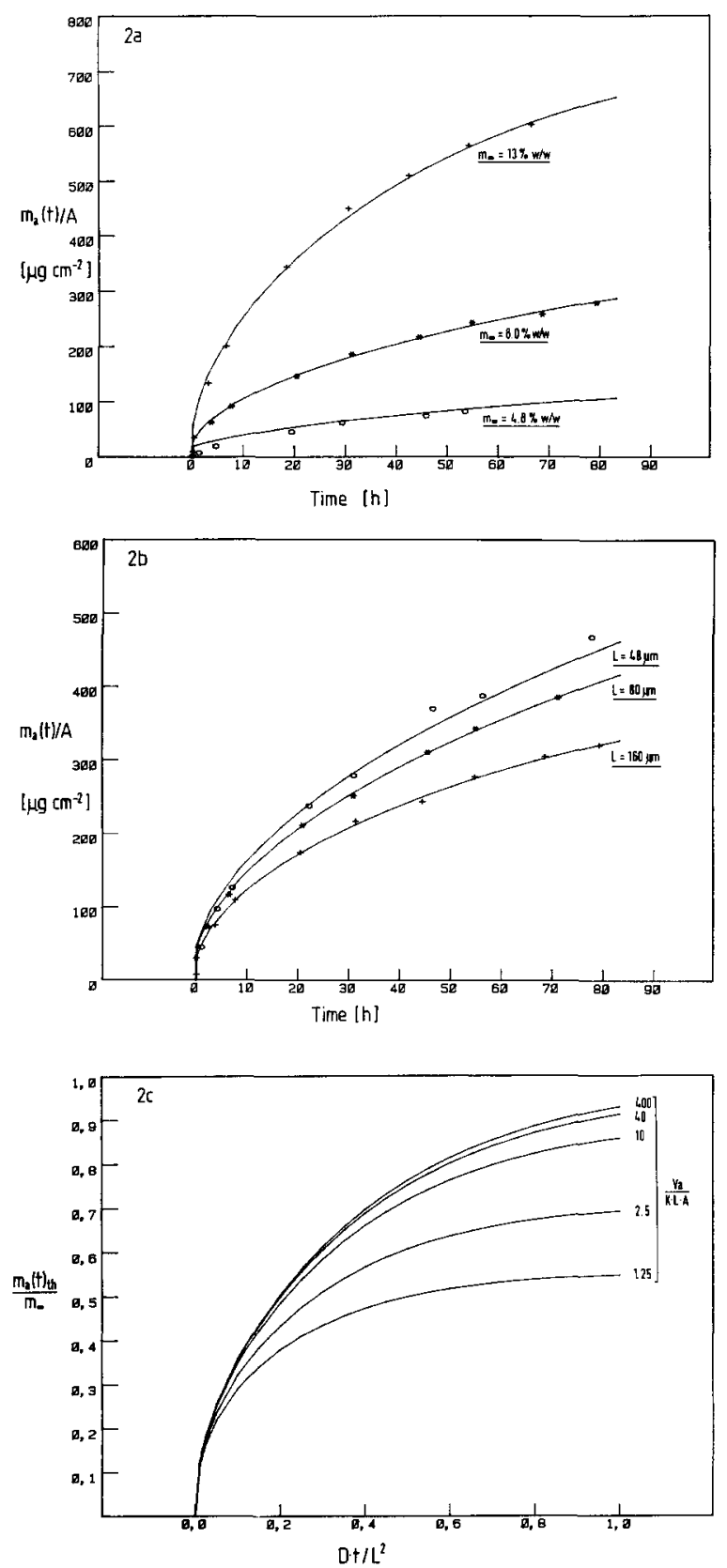

Fig. 2. Diffusion of clenbuterol out of polymer matrix. (a) Effect of drug loading ( $m_{\infty}$ ) on least-squares fits of theoretical $m_{\mathrm{a}}(t)_{\text {th }}$ values (continuous lines) to experimentally determined coordinates of $m_{\mathrm{a}}(t)$ exp; (b) effect of matrix thickness $(L)$ on least-squares fits of theoretical $m_{\mathrm{a}}(t)_{\mathrm{th}}$ values (continuous lines) to experimentally determined coordinates of $m_{\mathrm{a}}(t)_{\text {exp }}$; (c) dimensionless plots of $m_{\mathrm{a}}(t)_{\mathrm{th}} / m_{\infty}$ vs $D t / L^{2}$ as a function of the dimensionless variable $V_{\mathrm{a}} / K L A$.
TABLE 2

Diffusion of clenbuterol out of a polymer matrix

\begin{tabular}{llll}
\hline $\begin{array}{l}\text { Matrix } \\
\text { thickness } \\
(\mu \mathrm{m})\end{array}$ & $\begin{array}{l}\text { Drug } \\
\text { loading } \\
(\% \mathrm{w} / \mathrm{w})\end{array}$ & $\begin{array}{l}\text { Diffusivity } \\
\left(\times 10^{11}\right)\left(\mathrm{cm}^{2} \mathrm{~s}^{-1}\right)\end{array}$ & $\begin{array}{l}\text { Partition } \\
\text { coefficient }\end{array}$ \\
\hline 45.2 & 6 & $2.14(n=2)$ & 84.6 \\
45.2 & 8 & $2.57 \pm 0.405(n=8)$ & $17.0 \pm 48.1$ \\
45.2 & 11 & $3.89 \pm 2.29(n=4)$ & $83.8 \pm 76.2$ \\
45.2 & 13 & $6.75 \pm 5.09(n=6)$ & $100 \pm 169$ \\
23.1 & 8 & $5.92 \pm 0.41(n=4)$ & $\begin{array}{c}9.98 \times 10^{-8} \\
\pm 9.5 \times 10^{-8}\end{array}$ \\
160 & 8 & $4.09 \pm 0.724(n=4)$ & $0.924 \pm 1.85$ \\
\hline
\end{tabular}

2c. It can be shown from this figure that if $K$ has a value below approx. 10 it exerts practically no influence on $\mathrm{d} m_{\mathrm{a}}(t) / \mathrm{d} t$. The receding concentration profile of drug within the polymer matrix with time rapidly leads to low concentrations at the outer edge of the matrix (shown for sink-conditions in: Carlsaw and Jaeger, 1959a). Partition coefficients close to unity thus have little effect on the magnitude of $\mathrm{d} m_{\mathrm{a}}(t) / \mathrm{d} t$. This is exactly the case for clenbuterol, whose measured partition coefficient between hydrated Eudragit NE30D and water is 0.4 . The three-dimensional space used for the Nelder-Mead calculations will, therefore, be flat in the $K$-direction. Large changes in the fitted values of $K$ will have little effect on the residual sum of squares, making $K$ fluctuate greatly.

\section{Diffusion through excised, human stratum corneum}

Plots of $m_{\mathrm{a}}(t)$ vs $t$ for diffusion through a plane membrane under non-sink conditions are sigmoidal in shape, as can be observed even with a single non-sink condition at $x=L$ (Jenkins et al., 1970). The typical curves of best fit shown in Fig. 3a for diffusion through stratum corneum with non-sink conditions at both boundaries are, however, not obviously sigmoidal. This occurs because the points of inflexion of the three curves (as determined by setting the second derivative of the splined polynomial for each curve to zero) occur at 71,70 and $57 \mathrm{~h}$, respective to increasing drug concentration. Up to these times, only some $5 \%$ of the total amount of drug had diffused through the membrane. Table 3 shows the diffusivities calcu- 

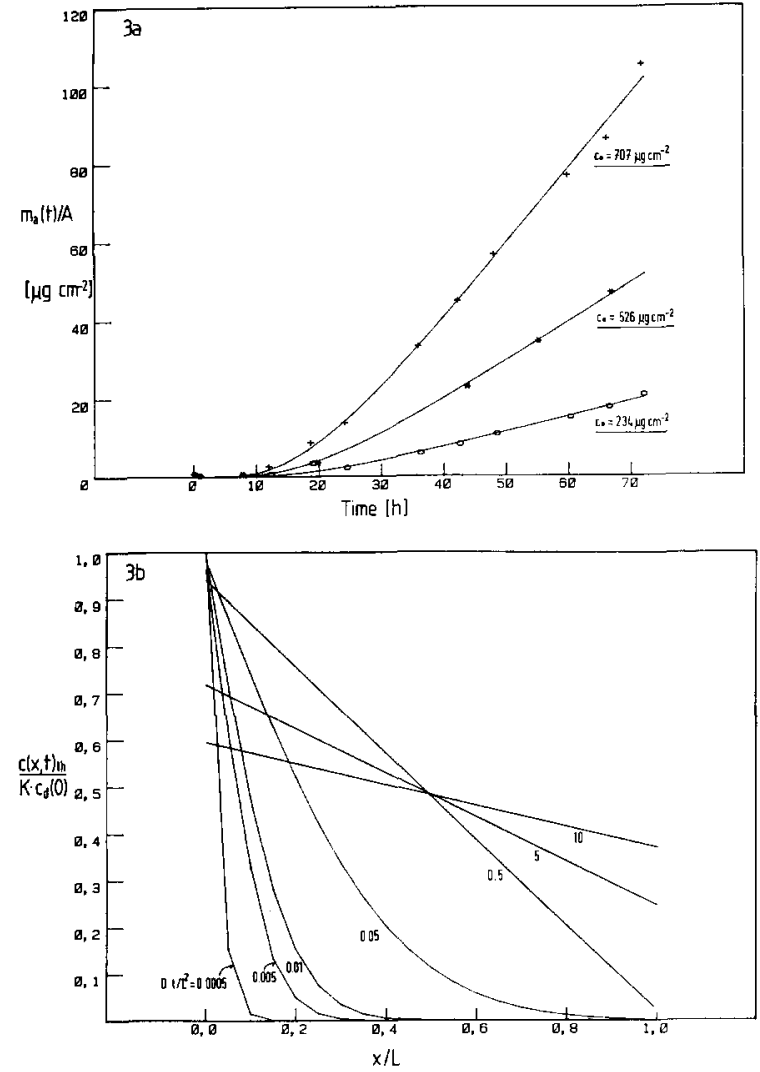

Fig. 3. Diffusion of clenbuterol through excised stratum corneum. (a) Effect of drug loading $\left(c_{0}\right)$ on least-squares fits of theoretical $m_{\mathrm{a}}(t)_{\text {th }}$ values (continuous lines) to experimentally determined coordinates of $m_{\mathrm{a}}(t)_{\text {exp }}$; (b) concentration profile within stratum corneum, $c(x, t)_{\mathrm{h}} / K c_{\mathrm{d}}(0)$ vs $t$, as a function of Dt $/ L^{2}$.

lated from six individual experiments on stratum corneum excised from the upper thigh of a single cadaver. The mean value of $3.97 \times 10^{-12} \pm 2.33 \times$
$10^{-12} \mathrm{~cm}^{2} \mathrm{~s}^{-1}$ is reasonable for drug molecules of this structure and size (Swarbrick et al., 1984). The standard deviation compares very favourably with literature data. To take a recent example, a diffusivity of $2.1 \times 10^{-5} \pm 2.9 \times 10^{-5} \mathrm{~cm}^{2} \mathrm{~h}^{-1}(n=6)$ was found for benzoic acid by fitting experimental data to an analytical solution for this problem (Parry et al., 1990). Error in the determination of $m_{\infty}$ is smaller and of much less importance here than with the polymer matrix, since the drug concentration in the donor solution can be directly measured with an error of $<1 \%$. The scatter seen in diffusivity within stratum corneum is due to the highly non-isotropic nature of this tissue. Much less variation is found when diffusivities are measured with the same method in an isotropic silicone membrane (Göpferich and Lee, 1990), with coefficients of variation of $<5 \%$ being obtained. By way of comparison Table 3 also includes for each curve the lag time $(\tau)$ as measured by extrapolation of the tangent to the point of inflexion of the numerical curve of best fit. Diffusivity was calculated from $\tau$ using the appropriate lag-time relationship for sink conditons: $\tau=L^{2} / 6 D$ (Table 3). The mean valueof $3.34 \times 10^{-12} \pm 1.79 \times 10^{-12} \mathrm{~cm}^{2}$ $\mathrm{s}^{-1}$ so obtained does not, however, substantially differ from that obtained from the curve fitting.

The partition coefficients shown in Table 3 for this problem are reasonable in value and shown less scatter than those obtained for the polymer matrix. The mean value of all the results is $212 \pm$ 165. The better fitting of these values is due to the stronger influence of $K$ on the diffusion rate for this problem. The lipophilic nature of the stratum corneum gives rise to high drug concentrations in

TABLE 3

Diffusion of clenbuterol through excised human stratum corneum (individual experiments)

\begin{tabular}{llllll}
\hline $\begin{array}{l}\text { Membrane } \\
\text { thickness } \\
(L)(\mu \mathrm{m})\end{array}$ & $\begin{array}{l}\text { Donor } \\
\text { concentration } \\
\left(\mu \mathrm{g} \mathrm{cm}^{-3}\right)\end{array}$ & $\begin{array}{l}\text { Diffusivity } \\
\left(\times 10^{12}\right) \\
\left(\mathrm{cm}^{2} \mathrm{~s}^{-1}\right)\end{array}$ & $\begin{array}{l}\text { Partition } \\
\text { coefficient } \\
(K)\end{array}$ & $\begin{array}{l}\text { Lag } \\
\text { time } \\
(\tau)(\mathrm{h})\end{array}$ & $\begin{array}{l}\text { Diffusivity a } \\
\left(\times 10^{12}\right) \\
\left(\mathrm{cm}^{2} \mathrm{~s}^{-1}\right)\end{array}$ \\
\hline 9.00 & 234 & 1.55 & 306 & 22.9 & 1.64 \\
10.5 & 526 & 2.17 & 291 & 20.6 & 2.48 \\
11.5 & 656 & 5.83 & 109 & 10.1 & 6.06 \\
12.0 & 671 & 6.08 & 70.8 & 13.0 & 5.13 \\
10.7 & 702 & 6.34 & 46.5 & 21.7 & 2.44 \\
9.50 & 708 & 1.87 & & 18.1 & 2.31
\end{tabular}

${ }^{\mathrm{a}} D^{\prime}=L^{2} / 6 \tau$. 
its outer edges and, large partition coefficients at $x=0$ and $x=L$. Fig. $3 b$ illustrates theoretically for release into a non-sink how the drug concentration profile within the stratum corneum changes with time. These profiles are quite different to those found for sink conditions (Barrer, 1951). The concentration directly adjacent to the donor solution, $c(0, t)$ is initially zero, but jumps at $t>0$ to a maximum value due to partitioning into this layer. Curved concentration profiles are seen which becomes more linear with time as $c(0, t)$ progressively decreases and $c(L, t)$ increases. High drug concentrations within the stratum corneum thus render $\mathrm{d} m_{\mathrm{a}}(t) / \mathrm{d} t$ sensitive to the magnitude of $K$. In contrast to the polymer matrix model, the multi-dimensional space used for the curve fitting procedure will, in this case, be highly contoured in the $K$-direction. The residual sum of squares is now sensitive to $K$, enabling a good fit of this parameter. Indeed, when isotropic, synthetic membranes are examined with this method much less variation is found than seen here with stratum corneum. For the diffusion of clenbuterol through the above-mentioned silicone membrane, for example, a coefficient of variation of $12 \%$ was obtained (Göpferich and Lee, 1990). These two contrasting examples of diffusion out of a polymer matrix and diffusion through stratum corneum thus illustrate well the contrasting restrictions imposed on the determination of $K$ from diffusional data.

\section{Diffusion out of polymer matrix through excised, human stratum corneum}

The effect of drug loading on the diffusion rate for the combined problem of diffusion out of a matrix through stratum corneum is shown in Fig. 4a. The curves are all sigmoid having an initial lag time due to diffusion through the stratum corneum. Furthermore, there is a simultaneous, continual decrease in diffusion rate of the drug out of the contiguous matrix with time, as illustrated by the receding theoretical concentration profile of the drug within the matrix for this bilayer system (Fig. 4b). This occurs at practically by the same rate as that seen with the matrix alone (cf. Carslaw and Jaeger, 1959a), indicating that the presence of the stratum corneum does not substantially hinder
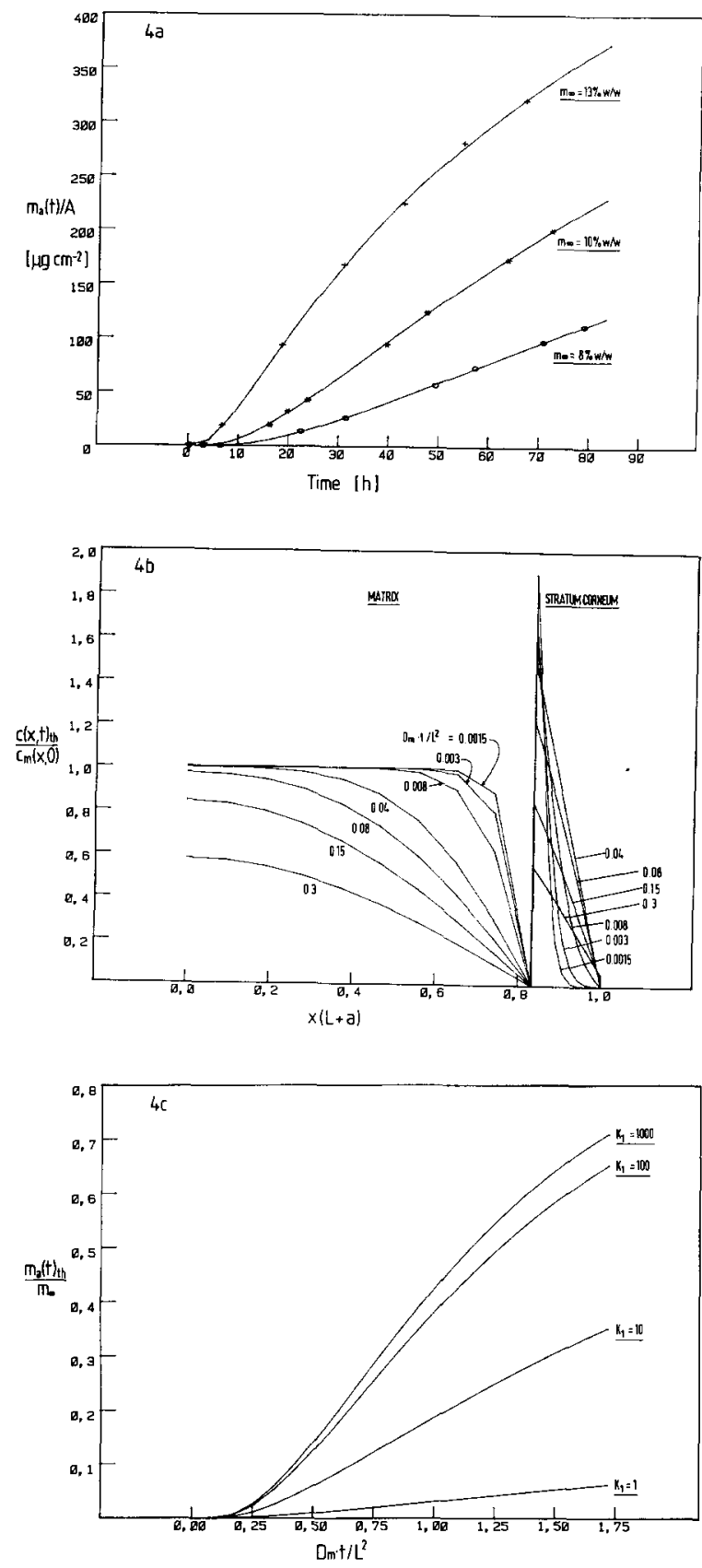

Fig. 4. Diffusion of clenbuterol out of a polymer matrix through excised stratum corneum. (a) Effect of drug loading $\left(m_{\infty}\right)$ on least-squares fits of theoretical $m_{\mathrm{a}}(t)_{\mathrm{th}}$ values (continuous lines) to experimentally determined coordinates of $m_{\mathrm{a}}(t)_{\text {exp }}$; (b) concentration profile within matrix and stratum corneum, $c(x, t)_{\mathrm{th}} / c_{\mathrm{m}}(x, 0)$ vs $x /(L+a)$, as a function of $D_{\mathrm{m}} t / L^{2}$ for $K_{1}=300$; (c) dimensionless plots of $m_{\mathrm{a}}(t)_{\mathrm{th}} / m_{\infty}$ vs $D_{\mathrm{m}} t / L^{2}$ as a function of $K_{1}$. 
TABLE 4

Diffusion of clenbuterol out of polymer matrix through excised human stratum corneum

\begin{tabular}{llll}
\hline $\begin{array}{l}\text { Matrix } \\
\text { thickness }(\mu \mathrm{m})\end{array}$ & $\begin{array}{l}\text { Drug loading } \\
(\% \mathrm{w} / \mathrm{w})\end{array}$ & $\begin{array}{l}\text { Diffusivity } \\
\left(\mathrm{cm}^{2} \mathrm{~s}^{-1}\right)\left(\times 10^{11}\right)\end{array}$ & Partition coefficient \\
\hline 50 & 8 & $D_{\mathrm{m}}=5.39 \pm 4.08(n=6)$ & $K_{1}=7.89 \times 10^{4} \pm 9.18 \times 10^{4}$ \\
& & $D_{\mathrm{s}}=0.178 \pm 0.034$ & $K_{2}=6.64 \times 10^{3} \pm 3.94 \times 10^{3}$ \\
50 & 10 & $D_{\mathrm{m}}=5.62 \pm 5.01(n=7)$ & $K_{1}=8.42 \times 10^{5} \pm 0.21 \times 10^{5}$ \\
& & $D_{\mathrm{s}}=0.246 \pm 0.145$ & $K_{2}=1.10 \times 10^{3} \pm 2.92 \times 10^{3}$ \\
50 & 13 & $D_{\mathrm{m}}=3.91 \pm 3.07(n=3)$ & $K_{1}=6.95 \times 10^{4} \pm 8.79 \times 10^{4}$ \\
& & $D_{\mathrm{s}}=0.536 \pm 0.115$ & $K_{2}=1.19 \times 10^{3} \pm 1.68 \times 10^{3}$ \\
\hline
\end{tabular}

diffusion out of the matrix, even though $D_{\mathrm{s}}$ is almost an order of magnitude lower than $D_{\mathrm{m}}$. This is evidently due to the large partition coefficient existing at the boundary between the polymer and lipophilic stratum corneum, which results in large amounts of drug being taken up into the stratum corneum at early times. Fig. $4 \mathrm{~b}$ shows how the solute concentration in the innermost region of the stratum corneum directly adjacent to the matrix, $c_{\mathrm{s}}(0, t)$, jumps at $t=0$ due to the immediate nature of the partitioning process. Thereafter $c_{\mathrm{s}}(0, t)$ declines with time and the concentration profiles within the stratum corneum become more linear as the drug diffuses slowly through this membrane.

The values obtained for $D_{\mathrm{m}}$ and $D_{\mathrm{s}}$ (Table 4) are comparable to those found for the matrix or stratum corneum alone. There is only one inconsistency: the $D_{\mathrm{m}}$ values do not show the dependence on drug loading seen before, whereas $D_{\mathrm{s}}$ now appear to be concentration dependent. The standard deviations are, however, large enough to make this observation questionable. Despite this scatter it is evident that the two diffusivities, which are an order of magnitude different in value, can be calculated from a single profile of $m_{\mathrm{a}}(t)_{\mathrm{th}}$ vs $t$ that is the best fit to experimental data.

As with the polymer matrix model it was not possible to obtain reasonable partition coefficients for the combined problem. The values in Table 4 are unacceptably large and show much scatter. This is due, once again, to the low drug concentrations existing at the outer edge of the matrix $(x=0)$ even after small times (Fig. 4b), which produces a highly non-linear influence of $K_{1}$ on the diffusion rate. Fig. $4 \mathrm{c}$ illustrates theoretically that $\mathrm{d} m_{\mathrm{a}}(t) / \mathrm{d} t$ hardly changes once $K_{1}$ exceeds 100 in magnitude. The value of $K_{1}$ for clenbuterol is, however, approx. 300, and will, therefore, lie on a very flat section of the fitting plane making its influence on the residual sum of squares negligible.

It thus appears possible to calculate diffusivities for the bilayer model, but not partition coefficients, by fitting the numerical solution to Fick's Second Law to experimental drug release data. A greater degree of scatter is, however, observed compared to measurements made on the two separate problems.

\section{Appendix}

For the combined problem as shown in Table 1 , a solution to the following system of equations is required:

$D_{\mathrm{m}} \cdot c_{\mathrm{m}}(x, t)_{x x}-c_{\mathrm{m}}(x, t)_{t}=0,-L<x<0, t>0$;

$D_{\mathrm{s}} \cdot c_{\mathrm{s}}(x, t)_{x x}-c_{\mathrm{s}}(x, t)_{t}=0,0<x<a, t>0$;

$K_{1} \cdot c_{\mathrm{s}}(0, t)-c_{\mathrm{m}}(0, t)=0, t>0 ;$

$K_{2} \cdot c_{\mathrm{a}}(t)-c_{\mathrm{s}}(a, t)=0, t>0$.

The method of Laplace transforms yields the following expression for $\mathscr{L}\left\{m_{\mathrm{a}}(t)\right\}(s)$ :

$$
\begin{aligned}
\mathscr{L} & \left\{m_{\mathrm{a}}(t)\right\}(s) \\
& =\left(c_{0} \sqrt{D_{\mathrm{m}}} b \sinh q_{\mathrm{m}} L\right)
\end{aligned}
$$




$$
\begin{aligned}
& \times\left(s \left\{\sqrt{D_{\mathrm{s}}}\left[b q_{\mathrm{s}} \cosh q_{\mathrm{s}} a+K_{2} \sinh q_{\mathrm{s}} a\right]\right.\right. \\
& \times \cosh q_{\mathrm{m}} L \\
& +K_{1} \sqrt{D_{\mathrm{m}}}\left[b q_{\mathrm{s}} \sinh q_{\mathrm{s}} a+K_{2} \cosh q_{\mathrm{s}} a\right] \\
& \left.\left.\times \sinh q_{\mathrm{m}} L\right\}\right)^{-1}
\end{aligned}
$$

where $q_{\mathrm{s}}=\sqrt{s} / \sqrt{D_{s}}$ and $q_{\mathrm{m}}=\sqrt{s} / \sqrt{D_{m}}$. Determination of the inverse transform using the residue theory is not straightforward. It could be approximated for short or long times (Guy and Hadgraft, 1980) or else inverted numerically (Addicks et al., 1989). Neither of these procedures appeared to us particularly advantageous compared to the direct numerical analysis of the complete problems, as presented in this paper.

\section{Acknowledgements}

We wish to thank Heidelberg University for providing funds for the purchase of a personal computer and also Boehringer Ingelheim for their generous financial support of this project. Thanks are also due to Professor H. Stricker, who kindly made some departmental funds available.

\section{References}

Addicks, W., Flynn, G., Weiner, N. and Curl, R., A mathematical model to describe drug release from thin topical applications. Int. J. Pharm., 56(3) (1989) 243-248.

Barrer, R.M., Diffusion in and through Solids, Cambridge University Press, London, 1951.
Carslaw, H. and Jaeger, J., Conduction of Heat in Solids, 2nd Edn, Clarendon, Oxford, 1959: (a) p. 98; (b) p. 17 et seq.

Crank, J., The Mathematics of Diffusion, 2nd Edn, Oxford University Press, London, 1975, pp. 56-59.

Crank, J. and Nicolson, P., A practical method for numerical evaluation of solutions of partial differential equations of the heat conduction type. Proc. Camb. Phil. Soc., 43 (1947) 50-67.

Göpferich, A. and Lee, G., The role of multi-dimensional diffusion in simple drug release/permeation experiments. Acta Pharm. Technol., 36 (1990) $27 \mathrm{~S}$.

Guy, R. and Hadgraft, J., A theoretical description relating skin permeation to the thickness of the applied medicament. Int. J. Pharm., 6 (1980) 321-332.

Guy, R., Hadgraft, J. and Maibach, H., A pharmacokinetic model for percutaneous absorption. Int. J. Pharm., 11 (1982) 119-129.

Jenkins, J., Nelson, P. and Spirer, L., Calculation of the transient diffusion of a gas through a solid membrane into a finite outflow volume. Trans. Faraday Soc., 66 (1970) 1391-1401.

Kligman, A. and Christophers, E., Preparation of isolated sheets of human stratum corneum. Arch Dermatol, 88 (1963) 702-710.

Nelder, J. and Mead, R., A simplex method for function minimisation. Comp. J., 10 (1967) 308-313.

Parry, G., Bunge, A., Silcox, G., Pershing, L. and Pershing, D., Percutaneous absorption of benzoic acid across human skin. I. In vitro experiments and mathematical modelling. Pharm. Res., 7 (1990) 230-236.

Spacek, P. and Kubin, M., Diffusion in gels. J. Polym. Sci., C16 (1967) 705-714.

Smith, G., Numerical Solution of Partial Differential Equations: Finite Difference Methods, 3rd Edn, Oxford University Press, Oxford, 1987, pp. 19-38.

Swarbrick, J., Lee, G., Brom, J. and Gensmantel, N., Drug permeation through human skin. II: Permeability of ionisable compounds. J. Pharm. Sci., 73 (1984) 1352-1355.

Zierenberg, B., Einsatz des Nelder-Mead-Verfahrens zur Optimierung der Freigabeparameter von Polymersystemen für Arzneistoffe. Acta Pharm. Technol., 30 (1985) 17-21. 